\title{
SOME UNCOMPLEMENTED UNIFORM ALGEBRAS
}

\section{ALAIN ETCHEBERRY}

ABSTRACT. The uniform algebras specified by the Wermer embedding theorem are not linearly homeomorphic to a complemented subspace of any Banach space $C(Y)$.

Let $A$ be a uniform algebra on a compact Hausdorff space $X$. Assume there is a character in the maximal ideal space $M(A)$, contained in a nontrivial Gleason part $P$ and admitting a unique representing measure $m$ on $X$.

THEOREM. $A$ is never linearly homeomorphic to a complemented subspace of a space $C(Y), Y$ a compact Hausdorff space.

RemarKs. Glicksberg [4] asked whether a closed nonselfadjoint subalgebra of $C(X)$ must be uncomplemented in $C(X)$, and answered this in the affirmative when $X$ is a compact group and $A$ is translation invariant. Rudin [9] had shown that the disc algebra is uncomplemented in $C(T)$ ( $T$ the unit circle) and that $H^{\infty}(d t)$ (dt Haar measure on $T$ ) is uncomplemented in $L^{\infty}(d t)$. Pełczyński [6] and Rosenthal [8] proved a theorem like the one in this paper for the disc algebra (Rosenthal's result is stated for algebras of generalized analytic functions). We will use their basic idea. Finally Pełczyñski [7] proved that $A$ is uncomplemented in $C(X)$ if the annihilator of $A$ is separable. This applies, in particular, to uniform algebras, such as $R(X)$, with at most a countable number of nontrivial Gleason parts and no completely singular annihilating measure in the sense of the F. and M. Riesz theorem [3, VI. 2.3 and VI. 3.2]. Our theorem applies, in particular, to Dirichlet algebras, logmodular algebras, the polydisc algebras $A\left(D^{n}\right)$, and algebras of generalized analytic functions (cf. [3, p. 169]), and is somewhat disjoint from the result of Pełczyński.

Proof. An injective Banach space is one for which all closed isomorphic embeddings into Banach spaces are complemented in these spaces. It is known [5] that complemented subspaces of injective spaces

Presented to the Society, April 14, 1973; received by the editors June 14, 1973.

AMS (MOS) subject classifications (1970). Primary 46J10, 46B05.

Key words and phrases. Uniform algebra, Gleason part, unique representing measure, F. and M. Riesz theorem, Wermer embedding theorem, uncomplemented subspace of $C(X)$, injective Banach space.

(c) American Mathematical Society 1974 
are injective and that $C(X)^{* *}$ (the second dual of $C(X)$ ) is an injective space. Thus, it is enough to prove that the second dual $A^{* *}$ of $A$ contains a complemented subspace linearly homeomorphic to the space $H^{\infty}(d t)$.

We prove first that $A^{* *}$ contains a complemented subspace isomorphic to $H^{\infty}(m)$, the $w^{*}$-closure of our algebra $A$ in the Banach space $L^{\infty}(m)$. By the Riesz representation theorem, the Lebesgue decomposition theorem and the Radon-Nikodym theorem, we have (up to isometric isomorphism), $C(X)^{*}=L^{1}(m) \oplus S, S$ being the closed subspace of all measures singular to $m$. By the F. and M. Riesz theorem, $A^{\perp}=A^{\circ} \oplus Z$, where $A^{\circ}$ is the part of the annihilator contained in $L^{1}(m)$ (this subspace can be identified (cf. [1, 4.2.12]), but that is irrelevant here), and $Z$ consists of those annihilating measures singular to $m$. Now $(\sim$ stands for "linearly homeomorphic to"),

$$
\begin{aligned}
A^{*} & =C(X)^{*} / A^{\perp} \sim\left(L^{1}(m) / A^{\circ}\right) \times(S / Z) \text { and } \\
A^{* *} & \sim\left(L^{1}(m) / A^{\circ}\right)^{*} \times(S / Z)^{*} .
\end{aligned}
$$

We have used here the fact that, for Banach spaces and closed subspaces, if $A=B \oplus C$ and $A^{\prime}=B^{\prime} \oplus C^{\prime}$, with $A^{\prime}, B^{\prime}, C^{\prime}$ subspaces of $A, B, C$, resp., we have $A / A^{\prime} \sim\left(B / B^{\prime}\right) \times\left(C / C^{\prime}\right)$ (consider the natural map). Observe also, in this general context, that $B / B^{\prime}$ is isomorphic to a complemented subspace of $A / A^{\prime}$ (the image of $B / B^{\prime}$ in $A / A^{\prime}$ under the natural map).

We claim that $\left(L^{1}(m) / A^{\circ}\right)^{*}=H^{\infty}(m)$. Indeed, identifying $A$ with a subspace of $L^{\infty}(m), A^{\circ}$ is precisely the (backward) polar of $A$, and $\left(L^{1}(m) / A^{\circ}\right)^{*}=A^{\circ \circ}$, the double polar of $A$. By the double polar theorem, this must be $H^{\infty}(m)$.

Finally we prove that $H^{\infty}(d t)$ (which we identity with $H^{\infty}(D)$, the algebra of all bounded analytic functions on the open disc $D$ ) is (isometrically) isomorphic to a complemented subspace of $H^{\infty}(m)$. The hypotheses of the theorem are precisely those of the Wermer embedding theorem [3, VI. 7.2 and V. 7.1] so, by that result, the Gleason part $P$ is an analytic disc and $H^{\infty}(P)$, the algebra of all bounded analytic functions on $P$ is (isometrically) isomorphic to $H^{\infty}(D)=H^{\infty}(d t)$. By a theorem of Gamelin ([2, Theorem 8.5], cf. also, [3, V. Example 6]), $H^{\infty}(P)$ is (isometrically) isomorphic to a complemented subspace of $H^{\infty}(m)$, a topological supplement being the ideal of those functions in $H^{\infty}(\mathrm{m})$ vanishing on $P$. This completes the proof.

ACKNOWLEDGEMENT. The author wishes to thank his supervisor Professor W. G. Badé for advice and encouragement.

\section{REFERENCES}

1. A. Browder, Introduction to function algebras, Benjamin, New York, 1969. MR 39 \#7431. 
2. T. Gamelin, Embedding Riemann surfaces in maximal ideal spaces, J. Functional Analysis 2 (1968), 123-146. MR 36 \#6941.

3. - Uniform algebras, Prentice-Hall, Englewood Cliffs, N.J., 1969.

4. I. Glicksberg, Some uncomplemented function algebras, Trans. Amer. Math. Soc. 111 (1964), 121-137. MR 28 \#4383.

5. D. B. Goodner, Projections in normed linear spaces, Trans. Amer. Math. Soc. 69 (1950), 89-108. MR 12, 266.

6. A. Pelczyński, On simultaneous extensions of continuous functions. A generalization of theorems of Rudin-Carleson and Bishop, Studia Math. 24 (1964), 285-304. MR 30 \#5184a.

7. - Uncomplemented function algebras with separable annihilators, Duke Math. J. 33 (1966), 605-612. MR 34 \#3373.

8. H. P. Rosenthal, Projections onto translation invariant subspaces of $L^{p}(G)$, Mem. Amer. Math. Soc. no. 63 (1966). MR 35 \#2080.

9. W. Rudin, Projection on invariant subspaces, Proc. Amer. Math. Soc. 13 (1962), 429-432. MR 25 \#1460.

Department of Mathematics, University of California, Berkeley, California 94720

Department of Mathematics, University of Chile, Casilla 653, Santiago, CHILE

Current address: Departamento de Matematicas, Universidad Simon Bolivar, Sartenejas, Baruta, Edo. Miranda, Apartoda Postal No. 5354, Venezuela 